\title{
Lower Limb Soft Tissue Necrotic Lesion
}

National Cancer Institute

\section{Source}

National Cancer Institute. Lower Limb Soft Tissue Necrotic Lesion. NCI Thesaurus. Code C78620

A necrotic process affecting the soft tissues of the lower extremity. 